\title{
Vision-Based Fall Detection System for Improving Safety of Elderly People
}

\author{
Fouzi Harrou ${ }^{\mathrm{a}}$, Nabil Zerrouki ${ }^{\mathrm{b}}$, Ying Sun ${ }^{\mathrm{a}}$, Amrane Houacine ${ }^{\mathrm{b}}$ \\ aKing Abdullah University of Science and Technology (KAUST) \\ Computer, Electrical and Mathematical Sciences and Engineering (CEMSE) Division, Thuwal, 23955-6900, Saudi Arabia \\ bUniversity of Sciences and Technology Houari Boumédienne (USTHB), LCPTS, Faculty of Electronics and Computer \\ Science, Algiers, Algeria
}

\section{Introduction}

Recognition of human movements is very useful for several applications, such as smart rooms, interactive virtual reality systems, human detection and environment modeling. The objective of this work focuses on the detection and classification of falls based on variations in human silhouette shape, a key challenge in computer vision. Falls are a major health concern, specifically for the elderly. In this study, the detection is achieved with multivariate exponentially weighted moving average (MEWMA) monitoring scheme, which is effective in detecting falls because it is sensitive to small changes. Unfortunately, a MEWMA statistic fails to differentiate real falls from some like-fall gestures. To remedy this limitation, a classification stage based on support vector machine is applied on detected sequences. To validate this methodology, two fall detection datasets have been tested: the University of Rzeszow fall detection dataset (URFD) and the fall detection dataset (FDD). The results of the MEWMAbased SVM are compared with three other classifiers: neural network, naïve Bayes and Knearest neighbor. These results show the capability of the developed strategy to distinguish fall events, suggesting that it can early raise an alert in the fall incidents.

Falls by elderly people can cause serious injury or death if sufferers remain on the ground for too long. Falls detection field has recently received increasing attention especially due to the fast up growth of communication and video monitoring systems. Several studies showed that falls are main reason of trauma for people with special needs like seniors. As reported by the World Health Organization in [1] 30\% of population over 65 years old experienced at least one trauma due to falls per year. Furthermore, $47 \%$ of seniors who have fallen lose their autonomies [2].

During last years, increased attention to fall detection has led to the development of several fall detection approaches [3, 4]. Some methods are generally based on information gathered by sensors like vibration and acceleration sensors [3, 4]. These methods use vibrations, sound, and human movements in fall detection $[4,5,6]$. However, these methods present several problems 
which limit their performance by increasing the rate of false detections. The acoustic sensors can be easily affected by noise. Furthermore, the detection using floor vibration sensors is limited only on surfaces equipped with sensors. The cost of the sensor installation on a large area of ground is very important, which makes these methods inappropriate in real life. In nowadays, small sensors are available and embedded is many daily devices such as in smartphones and smartwatches.

Other approaches focused in using information extracted from video sequences for fall detection [7]. These approaches have been proposed using a single camera, multiple cameras [8], omnidirectional ones [9] and stereo-pair cameras [10]. Indeed, a camera provides more information about the motion of the monitored person and attempts to extract important features from the video sequences to detect potential falls. In other words, the data collected by cameras are richer and more informative than standard sensors. In particular, vision-based fall detection can be useful inside hospital rooms and care facilities to detect falls and improve patient safety. Fall of patients in hospitals can lead to physical and emotional injuries, an extension of the period of stay, an increase of health care costs, and a demand for more hospital resources.

In this paper, we consider fall detection issue as an anomaly detection problem using MEWMA monitoring scheme [11]. The advantage of using the MEWMA chart is twofold. First, the use of MEWMA chart offers a better sensitivity in detecting falls. Second, this monitoring scheme is easy to implement in real time due to its low-computational cost. Unfortunately, the MEWMA monitoring chart cannot separate real falls from some like fall actions. This misclassification is due to the similarity between features of such gesture. To bypass this confusion, an SVM algorithm is applied on detected cases to classify the type of fall and reduce the false alarm rate. The choice of SVM is motivated by its high performance, in generalization in which the SVM can be adjusted to perform as linear or nonlinear algorithm by using nonlinear kernels. Of course, the combination of MEWMA-SVM presents a high accuracy with a reduced computational time compared to that obtained via the conventional approaches.

The following Section presents vision based strategy, including human body extraction, image quality enhancement and feature generation. Section 3 reviews how anomaly detection is achieved by MEWMA chart. Then in Section 4, the SVM technique that distinguishes true falls from other fall-like actions is presented. In Section 5, we assess the performance of the developed approach. Finally, Section 6 concludes with a discussion and suggestions for future research directions. 


\section{A vision-based fall detection and classification strategy}

The fall-detection procedure is implemented in five main stages: data acquisition, image segmentation, feature extraction, fall detection, and fall classification (Fig. 1).
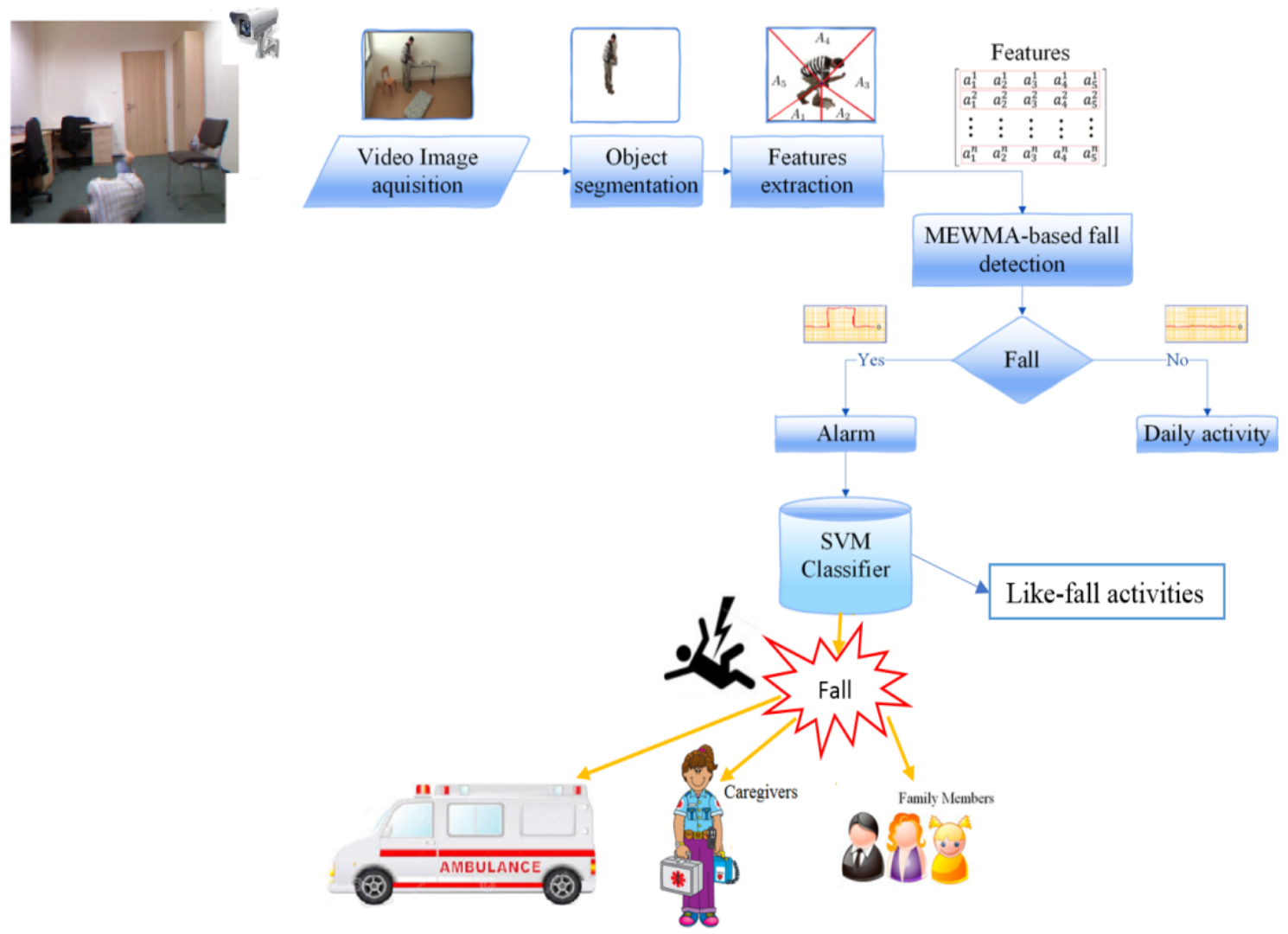

Fig.1. Schematic of the proposed strategy.

The data acquisition module consists of acquiring frames or video sequences from the camera. The segmentation module extracts the body silhouette from the background. Feature extraction is central to video-based fall detection. It can be defined as the process by which important discriminative information are extracted from a segmented body.

A segmentation is applied using background subtraction approach as illustrated in Fig. 2. The background template and the input images are respectively represented by Fig. 2 (a) and (b). Fig. 2 (c) and (d) illustrates the segmentation results (before and after the application of morphological operators).

(a)

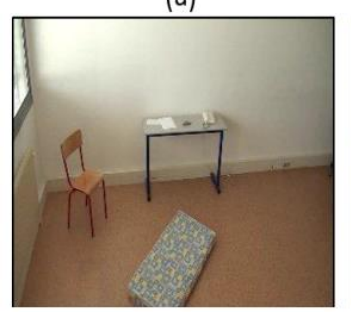

(b)

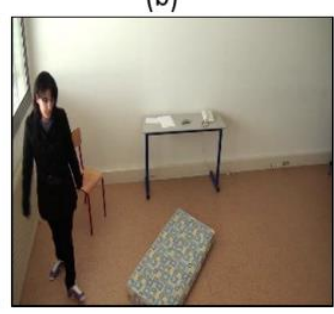

(c)

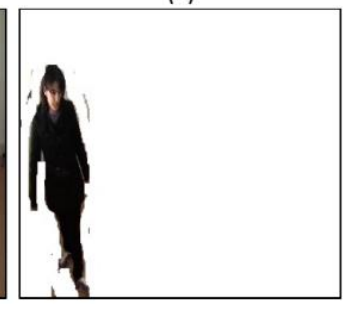

(d)

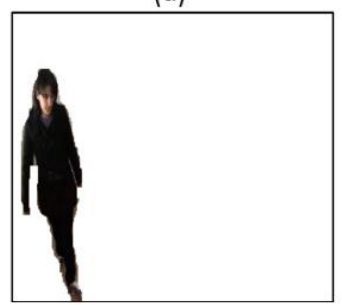


Fig.2. An example of background subtraction procedure.

For feature extraction phase, several characteristics have been used until now. However, most of them are highly dependent on the silhouette size and position. Body size changes when the distance from individuals to camera varies. A scaling operation with distance factor is frequently used, but this operation requires a prior camera calibration. For that reason, we propose the use of five areas constituting the human body. These areas are obtained by defining five lines from the silhouette's center of gravity (see Fig. 3). The first line is vertical, two other lines are situated at $45^{\circ}$ on each side from the first line, where the remaining segments are then traced at $100^{\circ}$ on each side of the two later lines (see Fig.3). Then, we normalize each area value by dividing its value by the whole area. Since video is assimilated to a sequence of frames, the area ratios extracted from the area ratios corresponding to each image are concatenated to constitute the whole vector.

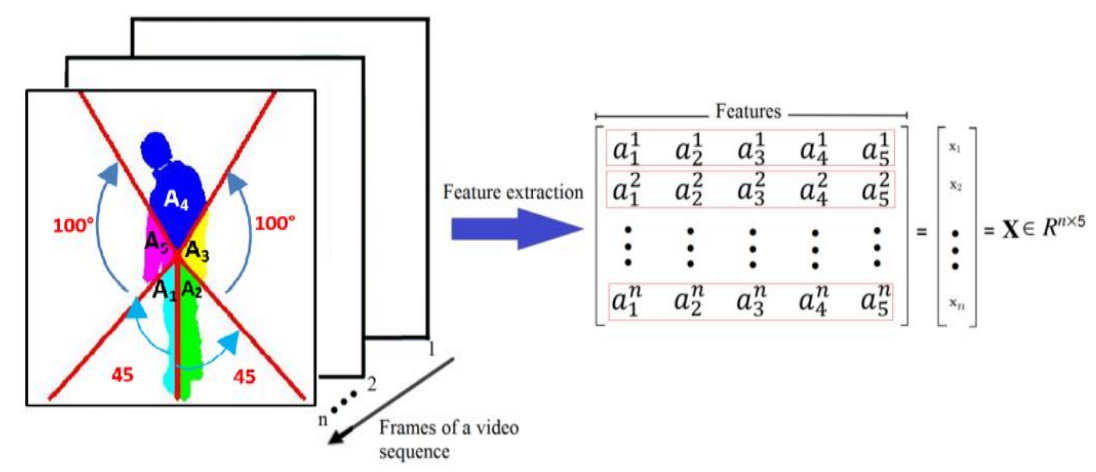

Fig.3. Extracted features used as input for fall detection.

\section{A MEWMA-based Fall Detection Strategy}

MEWMA chart has been widely used to monitor industrial processes for many years [12]. This chart takes the correlations between variables into account and monitor a set of cross-correlated variables simultaneously. MEWMA chart is able to detect small shifts, since the MEWMA statistic is a time-weighted average of all previous observations [12]. Let $X_{t}=\left(X_{1}, X_{2}, \ldots X_{m}\right)^{T}$ be a dataset obtained from a p-dimensional process. Herein, $\mathrm{p}$, represents the number of ratios that are computed for each image (i.e., $\mathrm{p}=5$ ). The MEWMA charting statistic is computed at each observation time point as follows [11]:

$$
\begin{cases}\mathbf{Z}_{t}=\Lambda \mathbf{X}_{t}+\left(\mathbf{I}_{m \times m}-\Lambda\right) \mathbf{Z}_{t-1} & \text { if } t>0 \\ \mathbf{Z}_{0}=\mu_{0}, & \text { if } t=0\end{cases}
$$

where $\Lambda=\operatorname{diag}(\lambda 1, \lambda 2, \ldots, \lambda \mathrm{m})$ and $\lambda \mathrm{j} \in(0,1]$ is a smoothing index or weight factor for the $\mathrm{j}$-th component of $X$, for $j=1,2, \ldots, m$, and $\mathbf{I m} \times m$ denotes the identity matrix. We can see that if $\lambda$ is 
small, then more weight is assigned to past observations. Thus the chart is tuned to have efficiency for detecting small changes in the process mean. Moreover, if $\lambda$ is large, then more weight is assigned to the current observations, and the chart is more suitable for detecting large shifts [12]. The MEWMA monitoring statistic is:

$$
\mathbf{V}_{t}^{2}=\mathbf{Z}_{t}^{T} \Sigma_{\mathbf{Z}_{t}^{-1}}^{-\mathbf{Z}_{t}}
$$

where $\Sigma_{\mathrm{Zt}}$ is the covariance matrix of $\mathbf{Z}_{\mathrm{t}}$. Then, the MEWMA chart with the charting statistic $\boldsymbol{V}_{t}^{2}$ gives a signal of mean shift in cases when $\boldsymbol{V}_{t}^{2}$ overpass a decision threshold h. The threshold $\mathrm{h}$ can be determined via simulation for a given probability of false alarm [12].

We note that it is often the case that an anomaly detector such as the MEWMA chart can detect anomalies but cannot distinguish detected anomalies from one another. Specifically, one disadvantage of the MEWMA anomaly detector is the lack of ability to discriminate the detected fall from false falls (i.e., fall-like). In this paper, falls classification based on the SVM algorithm is further developed to resolve this dilemma.

\section{SVM-based fall classification}

The SVM formalism was initially proposed by Vapnik [13], and has been widely exploited in the fields of classification and detection. The principle of the SVM is to transform the feature's space into a higher dimensional space using a kernel function. This transformation allows the obtaining of data approximately linear, and then defining the optimal separating hyper plane in the transformed space to classify different samples [13]. In the present application, SVM algorithm was selected as classifier since it could be considered as linear or nonlinear classifier by the use of nonlinear kernels. Different kernel functions can be chosen during classification process. In this work three kernels were tested namely: linear, polynomial, and radial basis function.

- Linear: $K\left(x_{i}, x_{j}\right)=x_{i}, x_{j}$

- Polynomial: $K\left(x_{i}, x_{j}\right)=\left(x_{i}, x_{j}+1\right)^{d}$, where $d$ is the degree of polynomial kernel.

- Radial basis function (RBF): $\mathrm{K}\left(x_{i}, x_{j}\right)=\exp \left(-\frac{\|x i,-x j\|^{2}}{2 \sigma^{2}}\right)$, where $\sigma$ is the width of Gaussian kernel.

In this work, SVM is applied to define the optimal separating hyperplane to classify detected sequences $y_{k} \in\{-1,+1\}$ into true falls and false falls, respectively. To assess the effectiveness of the proposed approach, a 3 fold-cross validation procedure is performed during classification, and different statistical measures were computed, one can cite the overall accuracy, F-measure, and the area under Receiver Operating Characteristic (ROC) [14]. 


\section{Experiments and Results}

\section{Data description}

The effectiveness of the developed strategy is validated using experimental data from two publically available databases: UR fall detection Database (URFD) [15] and Fall Detection Database (FDD) [16]. URFD consists of 70 videos with various actions performed in different ways. All sequences representing falls and normal activities have been recorded with RGB cameras comprising 30 frames per video. The second dataset FDD includes 191 videos with a rate of 25 images /s and a resolution of $320 \times 240$ pixels. The proposed combination of MEWMA-SVM is evaluated and compared with some powerful algorithms namely: KNN, neural network, and finally naïve Bayes classifier.

To quantify the efficiency of MEWMA chart, two metrics has been used: the false detection rate (FAR) and the miss detection rate (MDR). The FAR is the number of normal observations that are wrongly judged as anomaly (false alarms) over the total number of anomaly-free data. The MDR is the number of anomalies that are wrongly classified as normal (missed detections) over the total number of anomalies. The smaller the FAR and MDR are, the better the detection rate is.

The MEWMA monitoring chart is first performed through the training fall-free data. We selected a set of 300 sub-videos as the fall-free training data from only ordinary activity sequences. The features or descriptors extracted from the fall-free training data were arranged as a matrix $\mathrm{X}$ having 300 rows (number of frames) and 5 columns (five area ratios extracted from the posture of each image). These data were first scaled (to have a zero mean and a unit variance) and then utilized to train the MEWMA chart. When the MEWMA monitoring chart is applied using the fall-free data, the MEWMA threshold value is found to be $h=13.82$ for a false alarm probability of $\alpha=5 \%$ and $\lambda=0.2$.

\section{Detection results}

Firstly, the human body has been segmented and the area ratios have been extracted from the posture in the image. We studied two fall cases. In the first case, the testing sequence is tagged as real fall (case A), whereas the second sequence is tagged as false fall (like a fast lying (Case B)).

- Case study (A) - true fall:

Two examples are demonstrated in this section to illustrate the ability of the MEWMA scheme to identify fall events. In the first example, a fall occurred between frames 290 and 540 of the 
testing data. For the design of the MEWMA scheme, we chose $\lambda=0.2$ and threshold $h=13.82$. Fig. 4 (a) shows that MEWMA detected the fall event with some false alarm and missed detection (FAR=7.54\% and MDR=2\%). In the second example, MEWMA scheme with $\lambda=0.2$ is applied on testing data with fall between frame number 430 and 495 . Fig. 4 (b) shows MEWMA statistic clearly exceed the control limit, indicating the occurrence of abnormal event. The MEWMA chart detect this fall event but with some false alarms (i.e., FAR=11.16\%).

(a)

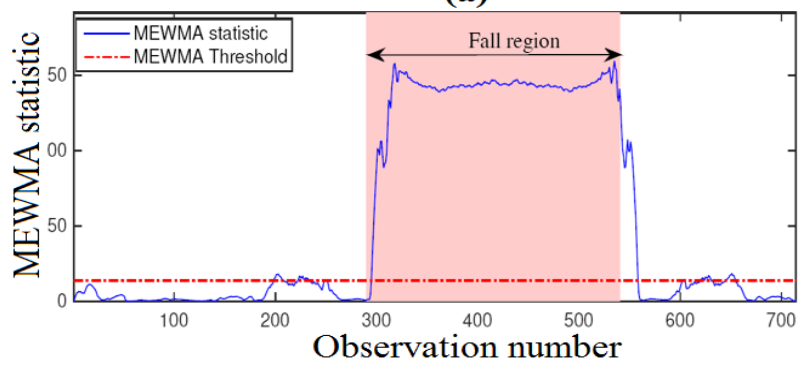

(b)

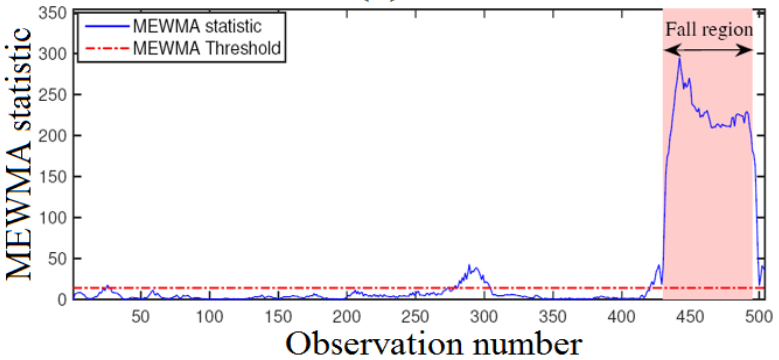

Fig.4. Results of MEWMA chart in the presence of a fall (a) first example, (b) second example.

- Case study (B): False fall - Lying down

In the second case study, lying down occurred between frames 180 and 500 of the testing data. Fig. 5 illustrates results of MEWMA detection. Several fall sequences are successfully detected, however there are some like fall activities were considered as falls. These confusions increase caused several false alarms in the detection system.

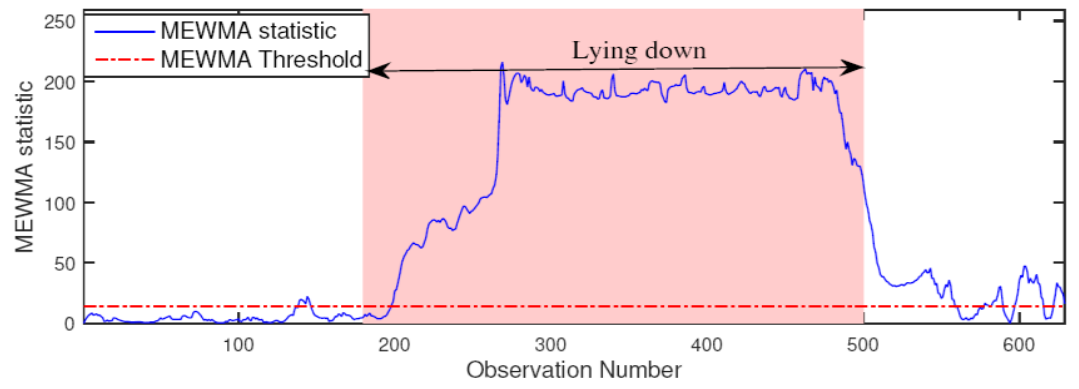

Fig.5. Results of the MEWMA chart in the presence of a false fall (Case B).

We note that the MEWMA chart cannot discriminate real falls from fall-like actions like lying down. To bypass this shortcoming a classification phase should be included after fall detection.

\section{Classification results}

- Data description

To assess the performance of the SVM classification, we selected only those sequences that were previously identified as falls (during the MEWMA detection stage). The sequences should be classified into one of the two classes namely true falls and false falls. These video samples 
were then split into training and testing sets. A three-fold cross validation procedure was used in this experiment.

\section{- Experimental Results}

Table 1 compares the combined MEWMA fall detector and SVM classifier (MEWMA-SVM) with some well-known classifiers such as: k-NN, neural network, and naïve Bayes, without detection step. It is worth noting that classifier's parameters have an important impact on the classification accuracy. Therefore, optimal parameters have been selected for each classifier corresponding to the highest accuracy. In the case of NN, we selected Multi-layer Perceptron (MLP) network with back propagation (BP) learning algorithm consisting of one hidden layer with fifteen neurons. For k-NN classification, the number of neighbors, $\mathrm{k}$, has been varied from 1 to 20 to determine the optimal value. The value of $\mathrm{k}$ providing the highest accuracy is 3 . For SVM classifier, different SVM-kernel parameters namely $\sigma$ and cost C; where $\sigma$ is the width of Gaussian kernel and $\mathrm{C}$ the parameter for the soft margin cost function, have iteratively tested. The pair providing the highest accuracy were selected $(\sigma=0.125$ and $\mathrm{C}=128)$.

Results shown in Table 1 testify that the MEWMA-SVM technique is more accurate than methods based only on classification alone. This fact is due to integrating the MEWMA detection phase, which separates daily activities from falling activities and thus reducing the number of sequences that will be used in SVM classification. Another reason that the MEWMA-SVM outperformed the neural network is that SVMs have a simple geometric representation and provide a sparse solution via structural risk minimization, while neural networks use empirical risk minimisation. MEWMA-SVM outperformed K-NN because of the capacity of SVM to train significant datasets, compared to the difficulty of k-NN in searching nearest neighbors for all frames. The MEWMA-SVM technique has also shown better performance than naïve Bayes classifier. This is relative to the independence assumption which is generally misappropriate in real world data. Furthermore, only detected sequences are classified by SVM, so just a part of videos is concerned by the classification task, which makes the processing much faster.

As shown in Table 1 (columns 9 and 10), the average training and testing times are calculated. The execution time is a significant tool to compare the complexity of algorithms. Neural network needs the longest training time to tune the parameters, while MEWMA-SVM obtains the shortest processing time. However, compared to K-NN, naïve Bayes and neural network, presents a reduced testing time. It is important to note that MEWMA-SVM combination 
remains significantly better, since it presents the highest accuracy and an acceptable processing time.

Table 1: Comparison between MEWMA-SVM and four commonly used classifiers.

\begin{tabular}{|c|c|c|c|c|c|c|c|c|c|}
\hline Classifier & Accuracy & Se & Sp & Precesion & Recall & $\begin{array}{c}\text { F- } \\
\text { measure }\end{array}$ & AUC & $\begin{array}{c}\text { Training } \\
\text { time }\end{array}$ & $\begin{array}{c}\text { Testing } \\
\text { time }\end{array}$ \\
\hline KNN & 91.94 & 1 & 0.86 & 0.838 & 1 & 0.91 & 0.93 & \multicolumn{2}{|c|}{0.28} \\
\hline $\begin{array}{c}\text { Neural } \\
\text { Network }\end{array}$ & 95.15 & 1 & 0.91 & 0.903 & 1 & 0.95 & 0.95 & 2.65 & 0.15 \\
\hline $\begin{array}{c}\text { Naïve- } \\
\text { Bayes }\end{array}$ & 93.55 & 1 & 0.886 & 0.87 & 1 & 0.93 & 0.94 & 0.61 & 0.26 \\
\hline $\begin{array}{c}\text { MEWMA- } \\
\text { SVM }\end{array}$ & 96.66 & 1 & 0.9493 & 0.9355 & 1 & 0.9524 & 0.9526 & 0.212 & 0.0357 \\
\hline
\end{tabular}

We also compared the proposed approach with existing techniques applied to FDD and URFD datasets (see Tables 2 and 3, respectively). Tables 2 and 3 illustrate a comparison between wellknown classifiers namely: KNN [15], and SVM [18], neural network [17], a variable-length particle swarm optimization (VPSO) neural network [19], and a directed acyclic graph SVM (DAGSVM) [2]. Results show that the MEWMA-SVM method outperformed other fall detection approaches and exhibited the highest accuracy. The main reason of the superiority of MEWMA-SVM fall detection method lies in the fact that the former uses the MEWMA chart, which is well reputed by its high sensitivity to anomaly. Instead the detection phase used in [18], where the decision is based on thresholds fixed manually. This superiority confirms also the advantage of the use of pixels-based area ratios which helped to a significant description of human body. Due to the translation and scaling invariant, their application is adapted to all video sequence types, and no prior camera calibration is required. Of course, based upon the results of the experiments carried out, the new method MEWMA-SVM seems to offer a more suitable fall detection capability than existing approaches.

Table 2: Comparison of different fall detection procedures using URFD database.

\begin{tabular}{|l|l|l|}
\hline & Approach & Overall accuracy (\%) \\
\hline B. Kwolek et al [18] & SVM & 90.00 \\
\hline \multirow{2}{*}{ Kwolek et al [15] } & SVM & 94.28 \\
\cline { 2 - 3 } & KNN & 95.71 \\
\hline The proposed approach & MEWMA-SVM & 96.66 \\
\hline
\end{tabular}

Table 3. Comparison of different fall detection procedures using FDD database

\begin{tabular}{|l|l|c|}
\hline & \multicolumn{1}{|c|}{ Approach } & Overall accuracy (\%) \\
\hline L. Alhimale et. Al,[17], & Neural Network & 94.27 \\
\hline X. Ma el al. [19] & VPSO-Neural Network & 92.17 \\
\hline M. Yu et al. [2] & DAGSVM & 96.09 \\
\hline The proposed approach & MEWMA-SVM & 97.02 \\
\hline
\end{tabular}




\section{Conclusion}

In summary, the results show that combining MEWMA with SVM was provide good separation of true falls from false falls in the higher-dimension features space via nonlinear mapping. Results recommend that the features corresponding to detected falls helps for improving the performance of true/false fall classification. Furthermore, the MEWMA monitoring has a central role in reducing the size of the features utilized as input data to SVM for classification. We also compared MEWMA-based SVM with SVM without the detection phase, neural network, K-nearest neighbor and naïve Bayes classifiers. The comparative results shows the superior classification capability of MEWMA-based SVM compared with other classifiers. There were several reasons that the SVM outperformed NN. Importantly, we found that the proposed features, detector and classifier are capable to detect and distinguish the type of fall with a higher accuracy compared to current techniques can. Of course, the results demonstrate that the MEWMA-based SVM improves fall classification.

In nowadays, many small sensors are available with the option to be embedded in many daily devices, such as smartphones and smartwatches, allowing free body movement for elderly. As future work, we plan to incorporate information from accelerometers or gyroscope with camera based approaches to improve further the detection accuracy. Moreover, we plan to incorporate more data inputs such as heart rate and blood pressure provided by a smartwatch or a smartphone to further enhance the effectiveness of a fall detection system.

\section{Acknowledgement}

The research reported in this publication was supported by funding from King Abdullah University of Science and Technology (KAUST), Office of Sponsored Research (OSR) under Award No: OSR-2015-CRG4-2582. The authors (Nabil Zerrouki and Amrane Houacine) would like to thank the LCPTS laboratory, Faculty of Electronics and Informatics, University of Sciences and Technology HOUARI BOUMEDIENE (USTHB) for the continued support during the research. We would like to thank the reviewers of this article for their insightful comments, which helped us to greatly improve its quality.

\section{References}

[1] Elgammal A, Harwood D, and Davis L. Non-parametric model for background subtraction. In Computer Vision-ECCV 2000, pages 751-767. Springer, 2000. 
[2] Yu M, Rhuma A, Naqvi S.M, Wang L, and Chambers J. A posture recognition-based fall detection system for monitoring an elderly person in a smart home environment. IEEE Transactions on Information Technology in Biomedicine, 16(6):1274- 1286, 2012.

[3] World Health Organization Ageing and Life Course Unit. WHO global report on falls prevention in older age. World Health Organization, 2008.

[4] San-Segundo R, Echeverry-Correa J.D, Salamea C, and Pardo J.M. Human activity monitoring based on hidden Markov models using a smartphone. IEEE Instrumentation \& Measurement Magazine, pages 27-31, 2016.

[5] Baek, J. and Yun, B.J. Posture monitoring system for context awareness in mobile computing. IEEE Transactions on instrumentation and measurement, 59(6), pp.1589-1599, 2010.

[6] Tao, Y. and Hu, H. A novel sensing and data fusion system for 3-D arm motion tracking in telerehabilitation. IEEE Transactions on Instrumentation and Measurement, 57(5), pp.1029-1040, 2008.

[7] Mubashir M, Shao L, and Seed L. A survey on fall detection: Principles and approaches. Neurocomputing, 100:144-152, 2013.

[8] Shieh W.Y and Huang J.C. Falling-incident detection and throughput enhancement in a multi-camera videosurveillance system. Medical engineering \& physics, 34(7):954-963, 2012.

[9] Miaou S-G, Sung P-H, and Huang C-Y. A customized human fall detection system using omni-camera images and personal information. In 1st Transdisciplinary Conference on Distributed Diagnosis and Home Healthcare, pages 39-42. IEEE, 2006.

[10] Jansen B and Deklerck R. Context aware inactivity recognition for visual fall detection. In Pervasive Health Conference and Workshops, pages 1-4. IEEE, 2006.

[11] Lowry C.A, Woodall W.H, Champ C.W, and Rigdon S.E. A multivariate exponentially weighted moving average control chart. Technometrics, 34(1):46-53, 1992.

[12] Montgomery D.C. Introduction to statistical quality control. John Wiley\& Sons, New York, 2005.

[13] Vapnik V. The nature of statistical learning theory. Springer Science \& Business Media, 2013.

[14] Zerrouki N and Houacine A. Automatic classification of human body postures based on curvelet transform. In Image Analysis and Recognition, pages 329-337. Springer, 2014.

[15] Kwolek B and Kepski M. Human fall detection on embedded platform using depth maps and wireless accelerometer. Computer methods and programs in biomedicine, 117(3):489-501, 2014.

[16] Charfi I, Miteran J, Dubois J, Atri M, and Tourkir. Definition and performance evaluation of a robust SVM based fall detection solution. In Eighth International Conference on Signal Image Technology and Internet Based Systems (SITIS), pages 218-224. IEEE, 2012.

[17] Alhimale L, Zedan H, and Al-Bayatti A. The implementation of an intelligent and video-based fall detection system using a neural network. Applied Soft Computing, 18:59-69, 2014.

[18] Kwolek B and Kepski M. Improving fall detection by the use of depth sensor and accelerometer. Neurocomputing, 168:637-645, 2015.

[19] Ma, X., Wang, H., Xue, B., Zhou, M., Ji, B., Li, Y., Depth-based human fall detection via shape features and improved extreme learning machine, IEEE journal of biomedical and health informatics, 18(6), 1915-1922, 2014.

Fouzi Harrou (fouzi.harrou@kaust.edu.sa) received the M.Sc. degree in Telecommunications and Networking from the University of Paris VI, in 2006 and the Ph.D. degree in Systems Optimization and Security in 2010 from the University Technology of Troyes (UTT), France. His current research interests include statistical decision theory and its applications, and fault detection and signal processing.

Nabil Zerrouki (nzerrouki@usthb.dz) received the engineer degrees and Master in Electrical Engineering from the University of Sciences and Technology Houari Boumédienne, Algiers, Algeria. 
His current research interests are computer vision, image processing and their applications to scene analysis and human-machine interaction.

Ying Sun (ying.sun @kaust.edu.sa) is an Assistant Professor of Statistics in the division of Computer, Electrical and Mathematical Sciences and Engineering (CEMSE) at King Abdullah University of Science and Technology (KAUST) in Saudi Arabia. She joined KAUST in June 2014 after one-year service as an assistant professor in the Department of Statistics at the Ohio State University, USA. Her research interests include spatio-temporal statistics with environmental applications, computational methods for large datasets, uncertainty quantification and visualization, functional data analysis, robust statistics, statistics of extremes.

Amrane Houacine (ahouacine@usthb.dz) is a professor at the Faculty of Electronics and Informatics, University of Sciences and Technology Houari Boumédienne, Algiers, Algeria. His current research interests include signal and image processing and their applications to human-machine interaction. 